\title{
101. Laplacien Local et la Décomposition de F. Riesz
}

\author{
Par Shin-ichi MATSUShItA
}

(Comm. by K. KUNUGI, M.J.A., July 12, 1956)

§1. Dans mes Notes précédentes, ${ }^{1)}$ j'exposai quelques résultats sur un théorème fondamental de $\mathrm{F}$. Riesz relatif à la décomposition de fonctions surharmoniques: les principales propositions établies dans ces Notes s'énoncent comme suit (sous quelque peu de corrections d'expression et typographiques).

Étant donné un domaine $D$ quelconque dans l'espace euclidien à $n$ dimensions $(n \geqq 3) E^{n}$, muni de la distance (euclidienne) $r(x, y)$, nous désignerons: $\mathfrak{M}^{+}(D)=$ cône convexe des mesures de Radon positives dans $D, \Gamma(D)=$ ensemble convexe constitué des fonctions surharmoniques, et $L^{+}(D)=$ ensemble convexe des fonctions $\geqq 0$ à supports compacts dans $D$. Désignons encore par $\mathfrak{R}(D), \Pi(D)$, et $L^{0}(D)$ enveloppes linéaires sur le corps réel de $\mathfrak{M}^{+}(D), \Gamma(D)$, et de $L^{+}(D)$ respectivement. †) $C^{p}$ désignera fonctions possédant des dérivées partielles continues jusqu'à l'ordre $p$ inclus, $1 \leqq p \leqq+\infty$.

Proposition 1 (Prop. $2[1]$ ). Pour toute $\mu \in \mathfrak{R}(D)$, on $a \phi_{D}\left(U^{\mu}\right)=\mu$.

Ici $\phi_{D}$ est une application linéaire de $\Pi(D)$ dans $\mathfrak{M}(D)$ définie par

$$
\left\langle\phi_{D}(f)\right\rangle(\varphi)=\int f(-\Delta \varphi) d x, \quad \Delta \varphi=\sum_{i=1}^{n} \frac{\partial^{2} \varphi}{\partial x_{i}^{2}}
$$

pour $f \in \Pi(D), \varphi \in L^{0}(D) \frown C^{2}$ ( $\phi_{D}$ est positive sur le produit $\Gamma(D)$ $\times\left(L^{+}(D) \frown C^{2}\right),{ }^{2)}$ et celui-ci étant positivement riche dans $L^{+}(D), \phi_{D}(f)$ peut se prolonger à une mesure positive tant que $\left.f \in L^{+}(D)\right),{ }^{3)}$ et $U^{\mu}$ désigne le potentiel newtonien:

$$
U^{\mu}(x)=\frac{\Gamma(n / 2)}{2(n-2) \pi^{n / 2}} \int r^{2-n}(x, y) d \mu(y) .
$$

Proposition 2 (Prop. $3[1]$ ). Pour que $f \in \Gamma(D) \quad(o u \in H(D) \equiv$ $\Gamma(D) \frown-\Gamma(D))$, il faut et il suffit que $\phi_{D}(f) \in \mathfrak{M}^{+}(D)\left(\right.$ resp. que $\phi_{D}(f)$ soit nulle).

Remarquons en passant que $H(D)$ est un espace vectoriel des fonctions harmoniques dans $D$.

1) S. Matsushita: Sur la décomposition de F. Riesz, I et II, C. R. Acad. Sci., Paris, 241, 1252-1254, 1373-1375 (1955), citées resp. [1] et [2].

$\dagger$ ) Tout élément $f$ de $\Pi(D)$ se représente en la forme $f=f_{1}-f_{2}$ pour $f_{1}, f_{2} \in \Gamma(D)$; si $f_{1}(x)=f_{2}(x)=+\infty$, il convient de définir que $f(x)=0$.

2) Prenons un voisinage compact du support de $\varphi \in L^{+}(D) \frown C^{2}$ comme $B$ dans Lemme du $\S 2$ et puis appliquons ce Lemme.

3) D'après une proposition de N. Bourbaki: Intégration, Livre 6, Prop. 2, 56 (1952). 
Proposition 3 (Thr. 1 [1]). Soit $D$ un domaine relativement compact, alors $\phi_{D}$ est un homomorphisme de $\Pi(D)$ sur $\mathfrak{M}(D)$, et le noyau de cet homomorphisme est $H(D)$.

Dans le quotient $\Pi(D) / H(D)$, i) chaque classe d'équivalence contient un et un seul potentiel $U^{\mu}$ d'une $\mu \in \mathfrak{M}(D)$, ii) la différence de deux fonctions quelconques de la même classe est harmonique dans $D$.

En d'autres termes, c'est la "décomposition de F. Riesz",

$$
f=U^{\phi_{D}(f)}+f_{D} \text {, où } f_{D} \in H(D) \text {, }
$$

dont la décomposition est uniquement déterminée.

Proposition 4 (Thr. $3[2]$ ). Pour que $f \in \Gamma(D), D$ étant quelconque, admette la décomposition (1.2), il faut et il suffit que $f \geqq u n e$ fonction convenable de $H(D)$.

§2. Dans la Note [1], il y a quelques arguments incomplets pour démonstration; nous exposons maintenant une démonstration complète pour Prop. 2 et rappelons la démonstration de Prop. 1 sous la forme plus adaptée dans ce qui suit. Avant de prouver ellesmêmes, nous montrons d'abord le

Lemme (Prop. $1[1]$ ). Soit $B$ un domaine relativement compact tel que $\bar{B} \subset D$, alors il existe pour chaque $f \in \Gamma(D)$ une suite des $f_{j} \in \Gamma(B) \frown C^{p}(p \geqq 2)$ telles que $f_{j} \nearrow f$ dans $B:^{4)}$ les mesures positives $\mu_{j}=\left(-\Delta f_{j}\right) d x_{B}$ converge vaguement vers $\left.\phi_{B}(f) .{ }^{5}\right)$

Comme $L^{+}(B) \frown C^{2}$ est positivement riche dans $L^{+}(B)$, il suffit de prouver pour $\varphi \in L^{+}(B) \frown C^{2}$. En effet, on a $(0 \leqq) \int \varphi d \mu_{j}=\int \varphi\left(-\Delta f_{j}\right) d x_{B}$ $=\int f_{j}(-\Delta \varphi) d x_{B}$ (formule de Green), qui converge vers $\int f(-\Delta \varphi) d x$ $=\left\langle\phi_{B}(f)\right\rangle(\varphi)$.

Démonstration de Prop. 1: Soient $\lambda$ et $\lambda^{\prime}$ deux mesures sphériques sur les sphères $\Sigma$ et $\Sigma^{\prime}$ de centre commun, $\Sigma \subset \Sigma^{\prime} \subset D{ }^{6)}$ Soit encore $\bar{B} \subset D$ et $\Sigma^{\prime} \subset B$, et appliquons le Lemme ci-dessus pour $U^{\mu}$ d'une $\mu \in \mathfrak{M}^{+}(D)$, en prenant $f_{j} \in \Gamma(B) \frown C^{3}$ telles que $f_{j} \nearrow U^{\mu}$ dans $B$. Comme $U^{\lambda-\lambda^{\prime}} \in L^{+}(B)$, on a alors $\int U^{\lambda-\lambda^{\prime}} d \phi_{D}(f)=\int U^{\lambda-\lambda^{\prime}} d \phi_{B}\left(U^{\mu}\right)=\lim _{j \rightarrow \infty} \int U^{\lambda-\lambda^{\prime}} d \mu_{j}$ $=\lim _{j \rightarrow \infty} \int U^{\mu_{j}} d\left(\lambda-\lambda^{\prime}\right)=\lim _{j \rightarrow \infty} \int f_{j} d\left(\lambda-\lambda^{\prime}\right)=\int U^{\mu} d\left(\lambda-\lambda^{\prime}\right)=\int U^{\lambda-\lambda^{\prime}} d \mu$, parce que $U^{\mu_{j}}-f_{j}$ est harmonique dans $B_{0}{ }^{7)}$ Les $U^{\lambda-\lambda^{\prime}}$ étant totaux dans $L^{0}(D)$, on en conclut que $\phi_{D}\left(U^{\mu}\right)=\mu$.

Démonstration de Prop. 2: Considérons une suite décroissante des sphères $\Sigma_{k} \subset \Sigma$ de centres communs telles que $\Sigma_{k} \rightarrow$ le centre $x_{0}$ de $\Sigma$ (et donc de toute $\Sigma_{k}$ ).

4) C'est un résultat classique: p. ex. voir T. Radó: Subharmonic Functions, Chelsea Publ. Co. (1949).

5) $d x_{B}$ désigne la restriction de $d x$ dans $B$.

6) $\lambda\left(\lambda^{\prime}\right)$ est la mesure de masse totale +1 réparties uniformément.

7) $\Delta\left(U^{\mu_{j}}-f_{j}\right)=\Delta f_{j}-\Delta f_{j}=0$, dont $\Delta f_{j} \in C^{1}$ dans $B$. 
Soit $f=f_{1}-f_{2}$ pour $f_{1}, f_{2} \in \Gamma(D)$ et supposons que $\phi_{D}(f) \geqq 0$ (par suite, $\phi_{B}(f) \geqq 0$ quel que soit $\left.B \subset D\right)$. Soit encore $\bar{B} \subset D, \Sigma \subset B$ et considérons $f_{j}^{p} \in \Gamma(B) \frown C^{3}$ telles que $f_{j}^{p} / f_{p}$ pour $p=1,2 . \quad \lambda_{k}$ étant sphérique sur $\Sigma_{k}$, on a alors $0 \leqq \int U^{\lambda_{k}-\lambda} d \phi_{B}(f)=\int U^{\lambda_{k}-\lambda}$ $d\left(\phi_{B}\left(f_{1}\right)-\phi_{B}\left(f_{2}\right)\right)=\lim _{j \rightarrow \infty} \int U^{\lambda_{k}-\lambda} d \mu_{j}^{1}-d \mu_{j}^{2}$ pour $\mu_{j}^{p}=\left(-\Delta f_{j}^{p}\right) d x_{B}$. D'ailleurs, $\lim _{j \rightarrow \infty} \int U^{\lambda_{k}-\lambda} d \mu_{j}^{p}=\lim _{j \rightarrow \infty} \int U_{j}^{p}\left(d \lambda_{k}-d \lambda\right)=\lim _{j \rightarrow \infty} \int f_{j}^{p}\left(d \lambda_{k}-d \lambda\right)=\int f_{p}\left(d \lambda_{k}-d \lambda\right)$ pour $p=1,2$, d'où $0 \leqq \int\left(f_{1}-f_{2}\right)\left(d \lambda_{k}-d \lambda\right)$ pour tout $k$. Si $f_{2}\left(x_{0}\right) \neq+\infty$, on a $f\left(x_{0}\right)-\int f d \lambda=\left(f_{1}\left(x_{0}\right)-\int f_{1} d \lambda\right)-\left(f_{2}\left(x_{0}\right)-\int f_{2} d \lambda\right)=\lim _{k \rightarrow \infty} \int f_{1}\left(d \lambda_{k}-d \lambda\right)-$ $\lim _{k \rightarrow \infty} \int f_{2}\left(d \lambda_{k}-d \lambda\right) \geqq 0$, e'est-à-dire $f\left(x_{0}\right) \geqq \int f d \lambda$ pour un point $x_{0}$ tel que $f_{2}\left(x_{0}\right) \neq+\infty$.

Cela étant, $f$ est presque-surharmonique au sens de SzpilrajnBrelot dans $D$ et $f=f_{0}$ presque partout (sur $D$ ) pour une et une seule $f_{0} \in \Gamma(D),{ }^{87}$ d'où $f_{0}+f_{2}=f_{1}$ presque partout, par suite, partout sur $D$; on en conclut que $f=f_{1}-f_{2}=f_{0} \in \Gamma(D)$.

Prop. 3 est une conséquence directe de Props. 1 et 2 .

§3. L'opérateur $\phi_{D}$ sera dit laplacien local (relatif à $D$ ); dans ce paragraphe nous faisons quelques remarques sur laplacien local. Comme on l'a vu au \$2, si $D$ est relativement compact, $\phi_{D}$ produit un homomorphisme de $\Pi(D)$ sur $\mathfrak{M}(D)$; on va prolonger cet opérateur pour un ensemble quelconque $X \subset E^{n}$. Tout d'abord, on indique que:

Proposition 5. Pour toute $\mu \in \mathfrak{M}\left(E^{n}\right)$, la mesure $\phi_{D}\left(U^{\mu}\right)$ est une restriction de $\mu$ à un ensemble $D$, notée $\pi_{J} \mu$;

Pour deux domaines $D_{1}, D_{2}$ tels que $D_{2} \subset D_{1}$, on a donc

$$
\phi_{D_{2}}(f)=\phi_{D_{1}}\left(U^{\phi_{D_{2}}(f)}\right)=\phi_{D_{2}}\left(U^{\phi_{D_{1}}(f)}\right) \text {, }
$$

quelle que soit $f \in \Pi\left(D_{1}\right)$. En particulier, posant $\phi=\phi_{D}$ pour $D=E^{n}$, on voit immédiatement que

$$
\phi_{D}(f)=\phi_{D}\left(U^{\phi(f)}\right)=\pi_{D} \cdot \phi(f) .
$$

D'une manière analogue, on peut définir en s'appuyant sur (3.3) l'opérateur $\phi_{X}$ pour un ensemble borélien $X$ quelconque par $\phi_{X}(f)=$ $\pi_{X} \cdot \phi(f), f \in \Pi\left(E^{n}\right)$, où $\pi_{X}$ désigne la restriction d'une mesure à $X$.

En posant $\phi_{X_{1}} \wedge \phi_{X_{2}}=\phi_{x_{1}}\left(U^{\phi} x_{2}{ }^{(f)}\right)$, on obtient aisément, $\phi_{X_{1}} \wedge \phi_{X_{2}}=$ $\phi_{X_{2}} \wedge \phi_{X_{1}}=\phi_{X_{1} \cap X_{2}}=\pi_{X_{1}} \phi_{X_{2}}=\pi_{X_{2}} \phi_{X_{1}}$ : l'ensemble de tous les $\phi_{X}$ constitue une algèbre commutative idempotente par rapport au produit $\Lambda$ et à la somme $\phi_{X_{1}} \dot{+} \phi_{X_{2}}=\phi_{X_{1}}+\phi_{X_{2}}-\phi_{X_{1}} \wedge \phi_{X_{2}}$, avec l'élément neutre $\phi$, qui est isomorphe à l'algèbre engendrée des opérateurs projectives dans $E^{n}$.

8) Voir p. ex. T. Radó: Loc. cit., p. 20; M. Brelot: Fonctions sous-harmoniques, presque sous-harmoniques ou sous-médianes, Ann. Univ. Grenoble, 21, 75-90 (1945). 
La norme de $\phi_{x}$ est définie par;

$$
\text { II } \phi_{X}\|\|=\sup _{\|j\| \leq 1}\left\|\phi_{X}(f)\right\| \text { pour } f \in \Gamma^{+}\left(E^{n}\right),{ }^{9)}
$$

où $\Gamma^{+}\left(E^{n}\right)$ est l'ensemble convexe des fonctions $\geqq 0$ de $\Gamma\left(E^{n}\right)$. En multipliant la valeur $2(n-2) \pi^{n / 2} / \Gamma(n / 2)$ à $\left\|\mid \phi_{X}\right\|$, c'est justement égale à la capacité d'un ensemble borélien $X$ (capacité selon de La Vallée Poussin). Les propriétés fondamentales de capacité résultent aisément de celles de la norme $\left\|\phi_{X}\right\|$; p. ex., $\|\mu\|=\left\|\phi_{X}\left(U^{\mu}\right)\right\| \leqq$ $\left\||| \phi_{X}\right\||\cdot| U^{\mu} \|$ pour $\mu \in \mathfrak{M}(X)$, d'où résulte que si $\left\|\left|\phi_{X}\right|\right\|=0$, il n'existe aucune mesure finie portée par $X$ telle qu'on ait $U^{\mu}<+\infty$.

Faisons finalement quelques remarques simples:

Remarque 1. Prenons au lieu du potentiel newtonien $U^{\mu}$ le potentiel $V^{\mu}$ dont le noyau est une fonction de Green $G(x, y)$ qui est régulière dans un domaine $D_{0} \supset D$ (domaine considéré):

$$
V^{\mu}(x)=\int G(x, y) d \mu(y)=U^{\mu}(x)-\int h(x, y) d \mu(y),
$$

où $h \in H\left(D_{0}\right)$ par rapport à $x \in D_{0}$. Alors on a la

Proposition 6. Les Propositions 1 5 restent vraies quand bien on remplacerait $U^{\mu}$ par $V^{\mu}$, quel que soit $D \subset D_{0}$.

Elle est une conséquence immédiate de ce que $\int h(x, y) d \mu(y)$ appartient aussi à $H\left(D_{0}\right)$ : C'est le cas où M. S. Hitotumatu a étudié dans leur travail récent. ${ }^{10)}$

Remarque 2. Dans la théorie des formes différentielles sur un variété (indéfiniment) différentiable, riemanien ou euclidien, l'opérateur de Laplace-Beltrami $\Delta_{0}=(d+\delta)^{2}=d \delta+\delta d$ joule le même rôle que notre $\phi$ pour une forme $\alpha$ de degré 0 (fonction ordinaire); en effet, pour toute forme $\beta$ de degré 0 , on a $\left(\beta, \Delta_{0} \alpha\right)=\int \beta\left(\Delta_{0} \alpha\right)^{*}=$

$$
\int \beta d \phi(\alpha)=\int \beta(-\Delta \alpha) e_{1 \cdots n} d x_{1} \cdots d x_{n},
$$

où $e_{1 \ldots n}$ est la composante du tenseur de Levi-Civita. ${ }^{11}$ Le potentiel de $\Delta_{0} \alpha$ relatif à la paramétrie $\omega(x, y)$ de MM. Knaster-Hodge s'exprime alors

$$
(\omega, \Delta \alpha)=\int \omega(x, y) d \phi(\alpha) .
$$

Remarque 3. Une distribution surharmonique $H$ se réduit à une fonction presque-surharmonique et celle-ci est encore égale à une et une seule fonction surharmonique presque partout sur $E^{n}$; d'autre

9) $\|\mu\|$ désigne la norme d'une mesure $\mu$; voir N. Bourbaki: Loc. cit.

10) S. Hitotumatu: Note on the Riesz decomposition of a subharmonic function, Comm. Math. Univ. Sancti Pauli, 3 (1955).

11) C'est égale à $\sqrt{g}, g$ étant le déterminant $\left\|g_{i j}\right\|$ des tenseurs covariants fondamentaux $g_{i j}$, en tant que le système de coordonnées soit positif; Cf. $p$. ex. W. V. D. Hodge: The Theory and Applications of Harmonic Integrals, Cambridge (1952). 
part, comme $-\Delta H$ est positive, elle n'est pas autre chose qu'une mesure positive. Ceci étant, le laplacien de M. L. Schwartz a le même sens que notre - $\phi$ et cela suggère que l'utilité de cet opérateur $\phi$, au lieu de $-\Delta$, fournit bien tant qu'il s'agit des distributions surharmoniques. ${ }^{12)}$

12) L. Schwartz: Théorie des Distributions, Hermann \& Cle, Paris (1950). C'est luj-même qui a donné une démonstration de la décomposition de F. Riesz sous la forme la plus élégante, mais leur méthode s'appuie essentiellement sur l'emploi de solution élémentaire d'équation différentielle pour distributions. 